\title{
Simulation of multi-layer THN/TIAIN thin film growth and calculation of its thermal conductivity
}

Pavel Moskovkin ${ }^{1}$, Jérôme Muller ${ }^{1}$, Stéphane Lucas ${ }^{1}$

1 University of Namur (LARN), rue de Bruxelles 61, 5000 Namur, Belgium

\section{Introduction}

Multi-layer titanium nitride/titanium aluminium nitride film growth was simulated by means of kinetic Monte Carlo code NASCAM [1]. Film growth was simulated for different number of bi-layers TiN/TiAIN, with the number of bi-layers equals from 5 to 100 . Results of the simulation were compared to experimental data [2]. It was shown that interfaces between individual layers make a significant contribution to a total thermal resistivity of the multi layer film.

Calculation of the thermal conductivity of simulated multi-layer film was conducted in two steps. Firstly, the thermal conductivity of the single layer was calculated by using Landauer relation based on effective medium theory [3]:

$k=\left[k_{p}\left(3 n_{p}-1\right)+k_{0}\left(2-3 n_{p}\right)+\left\{\left[k_{p}\left(3 n_{p}-1\right)+k_{0}\left(2-3 n_{p}\right)\right]^{2}+8 k_{0} k_{p}\right\}^{1 / 2}\right] / 4$

where $n_{p}$ and $k_{p}$ are porosity of the film and thermal conductivity of material which fills the pores, normally it equals to 0 . Interesting, that the relation is the same as Bruggeman's relation for effective dielectric constant.

Secondly, the thermal conductivity of the whole stack was calculated the following equation:

$\frac{1}{k}=\sum_{i=1}^{N} \frac{l_{i} / l}{k_{i}}+\sum_{j=1}^{N-1} r_{j}$

where $k_{i}$ is a thermal conductivity of a sub-layer " $i ", l_{i}$ is a thickness of the sub-layer " $i ", l$ is the total thickness of the layer, and $N$ is the number of sub-layers and $r_{j}$ are thermal resistivities of the interfaces between each TiN and TiAIN layers.
Firstly, it is necessary to setup NASCAM simulation for cases of multilayer deposition. To simulate such a film one has to simulate deposition $N=4 * n-1$ single layers. This number is easy to understand if one takes into account that the structure of the film is as follow:

TiN/Interface/TiAIN/interface/

TiN/interface/TiAIN/interface/

.../

TiN/interface/TiAIN/interface/

TiN/interface/TiAIN/

Therefore, for each bilayer it is necessary to simulate 4 single layers.

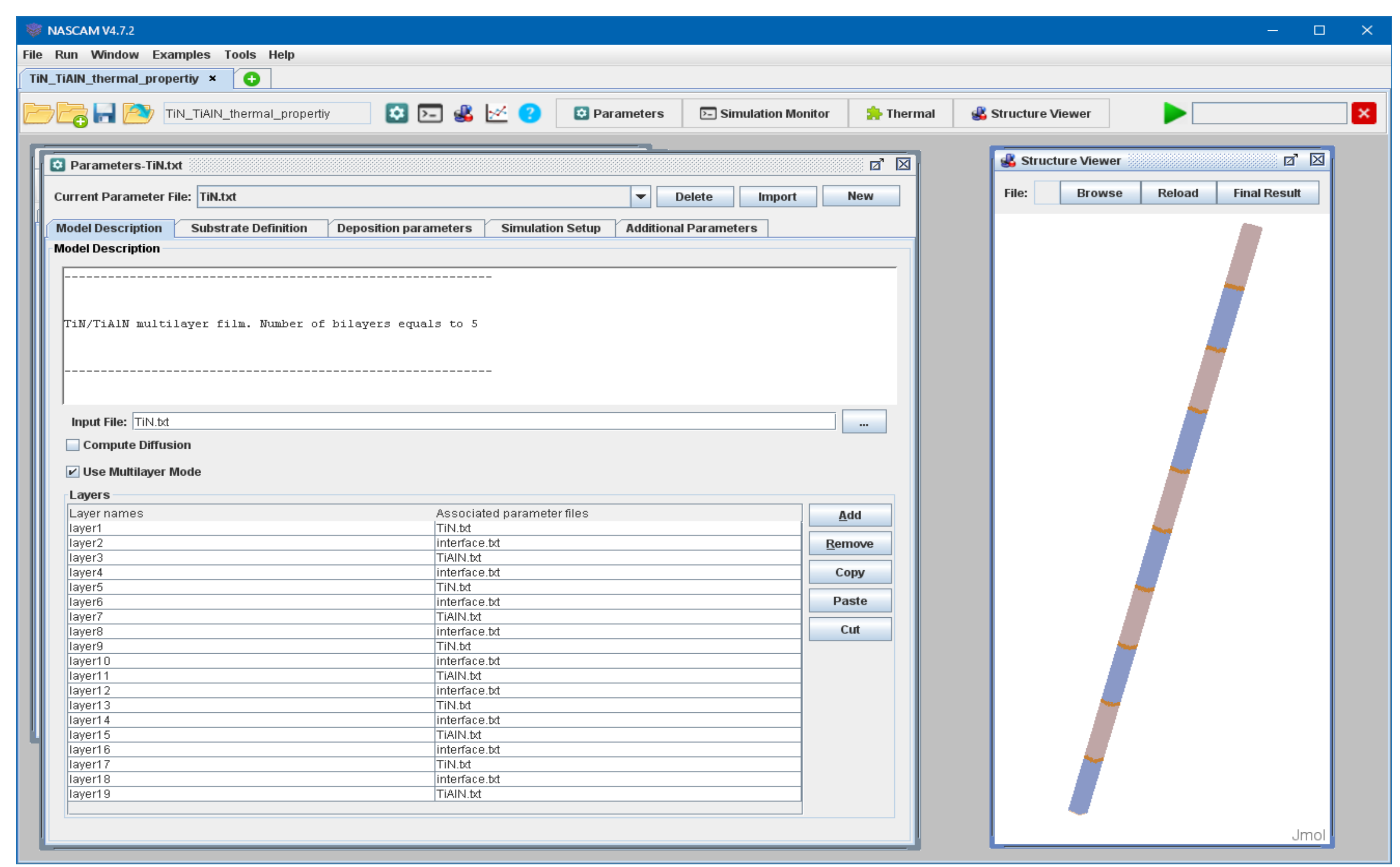

Simulation of deposition of 5 bilayers of TiN/TiAIN.

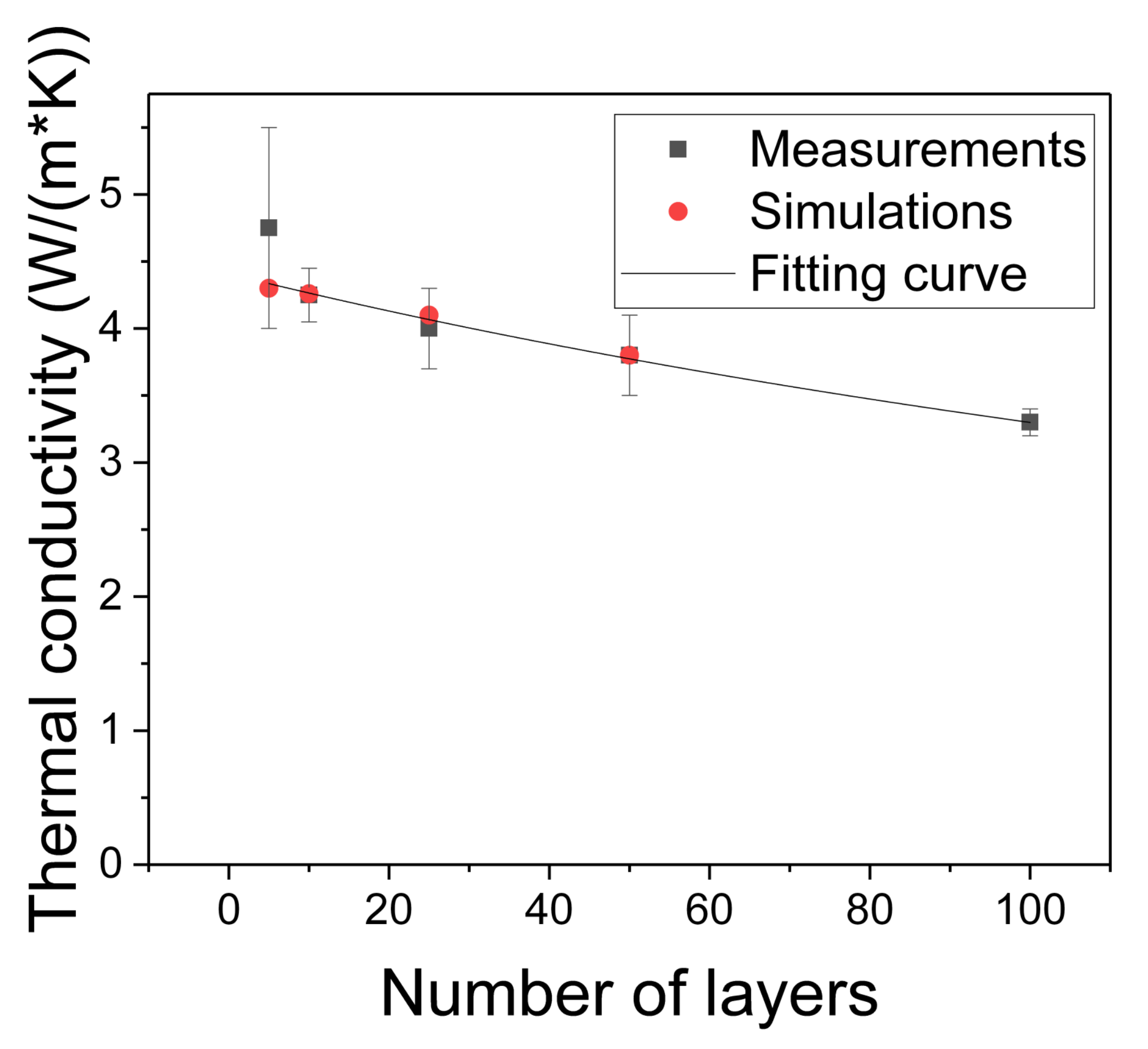

Experimental values of thermal conductivity of TiN/TiAIN multi layer film and values simulated by Thermal plugin and calculated by Eq. 2

\section{References}

[1] https://www.unamur.be/sciences/physique/ur/larn/logiciels/nascam

[2] M.K. Samani et al, Thermal conductivity of titanium nitride/titanium aluminum nitride multilayer coatings deposited by lateral rotating cathode arc, Thin Solid Films 578 (2015) 133-138).

[3] R. Landauer, The electrical resistance of binary metallic mixtures, J. Appl. Phys. 23, 779-784, 1952 